\title{
Chemical Constituents of Impatiens balsamina Stems and Their Biological Activities
}

\author{
Dong Hyun Kim ${ }^{1}$, Tae Hyun Lee ${ }^{1}$, Lalita Subedi ${ }^{2,3}$, Sun Yeou Kim ${ }^{2,3}$, and Kang Ro Lee ${ }^{1, *}$

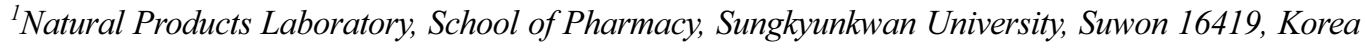 \\ ${ }^{2}$ Gachon Institute of Parmaceutical Science, Gachon University, 191 Hambakmoero, Yeonsu-gu, Incheon 21936, \\ Republic of Korea \\ ${ }^{3}$ College of pharmacy, Gachon University, 191 Hambakmoero, Yeonsu-gu, Incheon 21936, Republic of Korea
}

\begin{abstract}
The purification of the $\mathrm{MeOH}$ extract from Impatiens basamina by repeated column chromatography led to the isolation of one new tetrahydronaphthalene (1), together with eleven known compounds (2 - 12). The structure of the new compound (1) was determined by spectral data analysis $\left({ }^{1} \mathrm{H}\right.$ and ${ }^{13} \mathrm{C}-\mathrm{NMR},{ }^{1} \mathrm{H}-{ }^{1} \mathrm{H}$ COSY, HSQC, HMBC, NOESY, and HR-ESI-MS). Isolated compounds (1 - 12) were evaluated for their inhibitory effects on NO production in LPS-activated murine microglial BV-2 cells and their effects on NGF secretion from C6 glioma cells. Compounds 3, 7, and $\mathbf{1 0}$ reduced NO levels in LPS-activated murine microglial cells with $\mathrm{IC}_{50}$ values of $26.89,25.59$, and $44.21 \mu \mathrm{M}$, respectively. Compounds $\mathbf{1}, \mathbf{5}$, and 9 upregulated NGF secretion to $153.09 \pm 4.66,156.88 \pm 8.86$, and $157.34 \pm 3.30 \%$, respectively.
\end{abstract}

Keywords - Impatiens balsamina, balsaminaceae, tetrahydronaphthalene, neuroprotective effect

\section{Introduction}

Impatiens balsamina (Balsaminaceae), also known as Garden balsam, is widely distributed in Korea, China and India. I. balsamina has been used as Chinese medicine to treat anti-cancer and anti-inflammatory herb, also has been used as a Korean traditional medicine for the treatment of scrofulosis, carbuncle, and dysentery. ${ }^{1-3}$ Previous phytochemical investigations on I. balsamina reported the isolation of triterpenoid saponins, quinones, coumarin, flavonoids, and phenolic compounds, and there were several reports on various biological activities. ${ }^{4-10}$ Our earlier phytochemical investigation on white petal of I. balsamina resulted in the isolation of antineurodegenerative biflavonoid glycosides and antiinflammatory phenolic compounds. ${ }^{9,10}$ In a continuing search for bioactive constituents from Korean medical plant sources, we investigated the $\mathrm{MeOH}$ extract of stems of I. balsamina. The purification of hexane and EtOAc fractions by repeated column chromatography led to the isolation of one new tetrahydronaphthalene (1), together with eleven known compounds (2 - 12). The structure of 1 was established on spectral data analysis $\left({ }^{1} \mathrm{H}\right.$ and ${ }^{13} \mathrm{C}-\mathrm{NMR}$, ${ }^{1} \mathrm{H}-{ }^{1} \mathrm{H}$ COSY, HSQC, HMBC, and NOESY). Isolated com-

*Author for correspondence

Kang Ro Lee, Natural Products Laboratory, School of Pharmacy,

Sungkyunkwan University, Suwon 16419, Korea

Tel: +82-31-290-7710; E-mail: krlee@skku.ac.kr pounds (1 - 12) were evaluated for their inhibitory effects on nitric oxide (NO) production in lipopolysaccharide (LPS)-activated murine microglial cells and their effects on NGF secretion from C6 glioma cells.

\section{Experimental}

General experimental procedures - Optical rotations were measured on a Jasco P-2000 polarimeter using methanol solvent. Ultraviolet (UV) spectra were recorded with a Shimadzu UV-1601 UV-vis spectrometer. High Resolution ESI Mass Spectrometer data were obtained with a Waters SYNAPT G2 mass spectrometer. Infrared (IR) spectra were recorded on a Bruker IFS-66/S Fouriertransform IR spectrometer. NMR spectra were recorded on Varian UNITY INOVA 500 NMR spectrometer at 500 $\mathrm{MHz}\left({ }^{1} \mathrm{H}\right)$ and $125 \mathrm{MHz}\left({ }^{13} \mathrm{C}\right)$, and Bruker AVANCE III $700 \mathrm{NMR}$ spectrometer at $700 \mathrm{MHz}\left({ }^{1} \mathrm{H}\right)$ and $175 \mathrm{MHz}$ $\left({ }^{13} \mathrm{C}\right)$. Preparative HPLC was performed using a Gilson 321 pump with a Shodex Refractive Index Detector, YMC-Triart $\mathrm{C}_{18} 5 \mu \mathrm{m}$ column $(250 \times 10 \mathrm{~mm})$ and HAISIL 100 silica $5 \mu \mathrm{m}$ column $(250 \times 10 \mathrm{~mm})$. Silica gel 60 (Merck, 70 - 230 mesh and 230 - 400 mesh) and RP-C 18 silica gel (Merck, 230-400 mesh) was used for column chromatography. LPLC was performed over a LiChroprep Lobar-A silica 60 column (Merck, $240 \mathrm{~mm} \times 10 \mathrm{~mm}$ i.d.) equipped with a FMI QSY-0 pump. Merck precoated 
silica gel $\mathrm{F}_{254}$ plates and reversed-phase (RP)-18 $\mathrm{F}_{254 \mathrm{~s}}$ plates (Merck) were used for thin-layer chromatography (TLC). Spots were detected on TLC under UV light or by heating after spraying with anisaldehyde-sulfuric acid

Plant materials - The dried stems of I. balsamina $(2 \mathrm{~kg})$ were collected at Asan in Chungcheongnam-Do, Korea, in August 2014. The plants were identified by one of the authors (K.R.L.). A voucher specimen (SKKUNPL-1425) of the plant was deposited in the herbarium of the School of Pharmacy at Sungkyunkwan University, Suwon, Korea.

Extraction and isolation-The dried stems of $I$. balsamina $(2 \mathrm{~kg})$ were extracted with $80 \% \mathrm{MeOH}$ at room temperature. The filtrate was evaporated in vacuo to yield $\mathrm{MeOH}$ extract (294 g), which was suspended in distilled $\mathrm{H}_{2} \mathrm{O}(2.4 \mathrm{~L})$ and then successively partitioned with hexane, $\mathrm{CHCl}_{3}$, EtOAc and $n$-BuOH, yielding 20,11, 6, and $28 \mathrm{~g}$, respectively. The EtOAc fraction $(5 \mathrm{~g})$ was separated over a silica gel column with a solvent system of $\mathrm{CHCl}_{3} /$ $\mathrm{MeOH} /$ water (7:1:0.1) to yielded five sub-fractions (E1E5). Sub-fraction E1 (1.8 g) was chromatographed on a $\mathrm{RP}^{-\mathrm{C}_{18}}$ silica gel column with $30 \%$ aqueous $\mathrm{MeOH}$ to yield nine sub-fractions (E11-E19). Fraction E12 (430 $\mathrm{mg}$ ) was purified by $\mathrm{RP}^{-\mathrm{C}_{18}}$ semi-prep. HPLC (30\% $\mathrm{MeOH})$ to yield $2\left(9 \mathrm{mg}, t_{\mathrm{R}}=17.1 \mathrm{~min}\right)$. Fraction E2 $(1.1 \mathrm{~g})$ was separated by $\mathrm{RP}-\mathrm{C}_{18}$ silica gel column with $30 \%$ aqueous $\mathrm{MeOH}$ to give nine sub-fractions (E21E29). Sub-fraction E23 (161 mg) was purified using RP$\mathrm{C}_{18}$ semi-prep. HPLC $(30 \% \mathrm{MeOH})$ to yield $1(20 \mathrm{mg}$, $\left.t_{\mathrm{R}}=15.1 \mathrm{~min}\right), \mathbf{6}\left(24 \mathrm{mg}, t_{\mathrm{R}}=21.1 \mathrm{~min}\right)$, and $3(7 \mathrm{mg}$, $\left.t_{\mathrm{R}}=22.4 \mathrm{~min}\right)$. E3 $(557 \mathrm{mg})$ was subjected to $\mathrm{RP}-\mathrm{C}_{18}$ silica gel column with $45 \%$ aqueous $\mathrm{MeOH}$ to give five sub-fractions (E31-E35). Fraction E31 (148 mg) was purified by $\mathrm{RP}^{-\mathrm{C}_{18}}$ semi-prep. HPLC $\left(30 \% \mathrm{CH}_{3} \mathrm{CN}\right)$ to yield $4\left(100 \mathrm{mg}, t_{\mathrm{R}}=11.1 \mathrm{~min}\right)$. Fraction E5 $(235 \mathrm{mg})$ was purified using $\mathrm{RP}_{-} \mathrm{C}_{18}$ semi-prep. HPLC $\left(20 \% \mathrm{CH}_{3} \mathrm{CN}\right)$ to yield $5\left(32 \mathrm{mg}, t_{\mathrm{R}}=21.2 \mathrm{~min}\right)$. The hexane soluble fraction was chromatographed on a silica gel column with hexane/EtOAc (20:1 to $1: 1)$ to give eight sub-fractions (H1-H8). Fraction H2 (546 mg) was subjected to using a $\mathrm{RP}^{-\mathrm{C}_{18}}$ silica gel column with $95 \%$ aqueous $\mathrm{MeOH}$ to yield $11\left(9 \mathrm{mg}, t_{\mathrm{R}}=34.4 \mathrm{~min}\right)$. Fraction $\mathrm{H} 3(918 \mathrm{mg})$ was separated to $\mathrm{RP}-\mathrm{C}_{18}$ silica gel column with $90 \%$ aqueous $\mathrm{MeOH}$ to yield eight sub-fractions (H31-H38). Subfraction H32 $\left(85 \mathrm{mg}\right.$ ) was purified using $\mathrm{RP}^{-\mathrm{C}_{18}}$ semi-prep. HPLC $(90 \% \mathrm{MeOH})$ to yield $7\left(9 \mathrm{mg}, t_{\mathrm{R}}=36.9 \mathrm{~min}\right)$. Fraction $\mathrm{H} 36(117 \mathrm{mg})$ was purified by semi-prep. HPLC $\left(\mathrm{CHCl}_{3} /\right.$ $\mathrm{MeOH} 120: 1)$ to give $12\left(88 \mathrm{mg}, t_{\mathrm{R}}=11.0 \mathrm{~min}\right)$. Fraction $\mathrm{H} 5(2.4 \mathrm{~g})$ was chromatographed on a $\mathrm{RP}-\mathrm{C}_{18}$ silica gel column $(95 \%$ aqueous $\mathrm{MeOH})$ to give twelve sub-fractions (H51-H512). Subfraction H56 (89 mg) was purified using RP-C ${ }_{18}$ semi-prep. HPLC $(95 \% \mathrm{MeOH})$ to yield $8(4 \mathrm{mg}$, $\left.t_{\mathrm{R}}=17.8 \mathrm{~min}\right)$. Fraction H57 $(169 \mathrm{mg})$ was purified by semi-prep. HPLC (hexane/EtOAc 3:1) to yield 9 (3 mg, $\left.t_{\mathrm{R}}=15.3 \mathrm{~min}\right)$. Fraction $\mathrm{H} 512(112 \mathrm{mg})$ was purified using semi-prep. HPLC (hexane/EtOAc 3:1) to give 10 (7 mg, $t_{\mathrm{R}}=8.8 \mathrm{~min}$ ).

$1 \beta, 2 \alpha, 4 \beta$-Triol-1,2,3,4-tetrahydronaphthalene (1) Colorless gum. $[\alpha]_{\mathrm{D}}^{25}:-35.8(c$ 0.10, MeOH); IR (KBr) $v_{\max } \mathrm{cm}^{-1}: 3348,2938,2836,1448,1412,1055,1025$; UV $(\mathrm{MeOH}) \lambda_{\max }(\log \varepsilon) 201$ (0.75), 213 (0.45), 217 (0.38), $262(0.02) \mathrm{nm} ;{ }^{1} \mathrm{H}$ and ${ }^{13} \mathrm{C}$ NMR : see Table 1; HRESIMS $m / z 181.0865[\mathrm{M}+\mathrm{H}]^{+}$; (calcd. for $\mathrm{C}_{10} \mathrm{H}_{13} \mathrm{O}_{3}, 181.0865$ ).

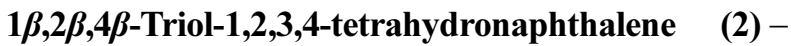
Colorless gum. $[\alpha]_{\mathrm{D}}^{25}:-57.8(c$ 0.11, MeOH); IR (KBr) $v_{\max } \mathrm{cm}^{-1}: 3340,2944,2832,1460,1408,1054,1021$; UV $(\mathrm{MeOH}) \lambda_{\max }(\log \varepsilon) 203$ (1.28), 211 (1.11), 215 (0.92), $256(0.04) \mathrm{nm} ;{ }^{1} \mathrm{H}$ and ${ }^{13} \mathrm{C}$ NMR : see Table 1.

Table 1. ${ }^{1} \mathrm{H}$ and ${ }^{13} \mathrm{C}$ NMR data of $\mathbf{1}$ and $\mathbf{2}$ in $\mathrm{CD}_{3} \mathrm{OD}$. ( $\delta$ in ppm, $500 \mathrm{MHz}$ for ${ }^{1} \mathrm{H}$ and $125 \mathrm{MHz}$ for $\left.{ }^{13} \mathrm{C}\right)^{a}$

\begin{tabular}{|c|c|c|c|c|}
\hline \multirow{2}{*}{ Position } & \multicolumn{2}{|l|}{1} & \multicolumn{2}{|l|}{2} \\
\hline & $\delta_{\mathrm{H}}$ & $\delta_{\mathrm{C}}$ & $\delta_{\mathrm{H}}$ & $\delta_{\mathrm{C}}$ \\
\hline 1 & $4.41 \mathrm{~d}(7.3)$ & 73.6 & $4.64 \mathrm{~d}(3.5)$ & 70.1 \\
\hline 2 & 4.09 ddd $(10.1,7.3,3.4)$ & 68.9 & $3.89 \mathrm{dt}(10.9,3.5)$ & 67.7 \\
\hline \multirow[t]{2}{*}{3} & 2.12 ddd $(13.5,10.1,4.7)$ & 37.1 & $2.15 \mathrm{ddd}(12.2,10.9,9.5)$ & 34.9 \\
\hline & $2.21 \mathrm{ddd}(13.5,4.7,3.4)$ & & 2.21 dddd $(12.2,6.1,3.5,1.0)$ & \\
\hline 4 & $4.89 \mathrm{~m}$ & 66.6 & $4.73 \mathrm{dd}(9.5,6.1)$ & 67.4 \\
\hline $4 a$ & & 137.5 & & 139.4 \\
\hline 5 & $7.41 \mathrm{dd}(7.3,1.5)$ & 128.1 & $7.57 \mathrm{~d}(7.5)$ & 126.7 \\
\hline 6 & $7.34 \operatorname{td}(7.3,1.5)$ & 127.1 & $7.30 \operatorname{td}(7.5,1.6)$ & 127.9 \\
\hline 7 & $7.29 \operatorname{td}(7.3,1.5)$ & 127.6 & $7.34 \operatorname{td}(7.5,1.6)$ & 127.3 \\
\hline 8 & $7.56 \mathrm{~d}(7.3)$ & 127.4 & $7.41 \mathrm{dd}(7.5,1.6)$ & 129.6 \\
\hline $8 \mathrm{a}$ & & 137.6 & & 135.9 \\
\hline
\end{tabular}

${ }^{a} J$ values are in parentheses and reported in $\mathrm{Hz}$; the assignments were based on ${ }^{1} \mathrm{H}-{ }^{1} \mathrm{H}$ COSY, HSQC, and $\mathrm{HMBC}$ experiments. 


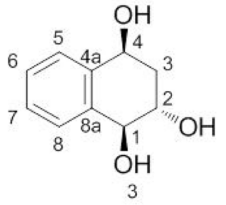

1<smiles>COc1c(-c2ccc(O)cc2)oc2cc(O)cc(O)c2c1=O</smiles>

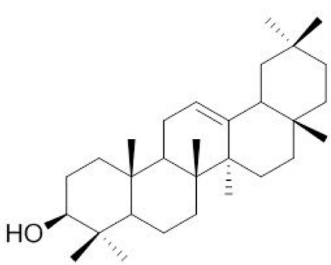

7

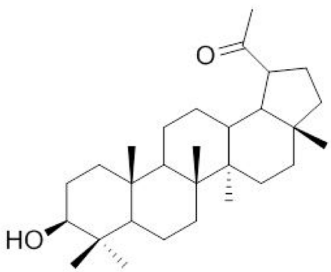

10<smiles>OC1CC(O)C(O)c2ccccc21</smiles><smiles>COc1cc(C2Oc3c(OC)cc(CCCO)cc3[C@@H]2COC(Cl)Cl)ccc1O</smiles>

3<smiles>O=C(O)c1ccc(O)cc1</smiles><smiles>CCC(C=C[C@@H](C)C1CCC2C3=CC[C@@H]4C[C@@H](O)CCC4(C)[C@H]3CC[C@]21C)C(C)C</smiles>

9

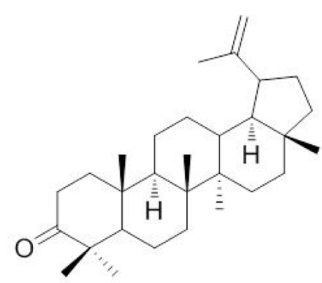

11

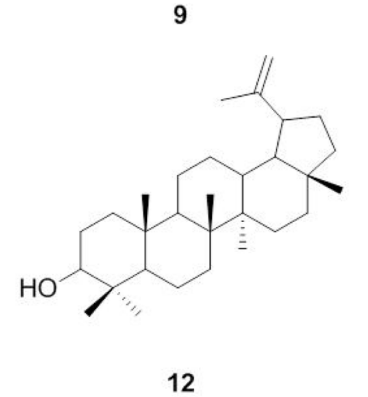

Fig. 1. Structures of compounds $\mathbf{1}$ - 12 isolated from I. balsamina

$(7 R, 8 S)$-Dihydrodehydrodiconiferyl alcohol-9- $\boldsymbol{\beta}-\boldsymbol{O}$-Dglucopyranoside (3) - White gum; ${ }^{1} \mathrm{H}$ NMR $\left(\mathrm{CD}_{3} \mathrm{OD}\right.$, $500 \mathrm{MHz}): \delta 6.99(1 \mathrm{H}, \mathrm{d}, J=1.9 \mathrm{~Hz}, \mathrm{H}-2), 6.85(1 \mathrm{H}, \mathrm{dd}$, $J=8.1,1.9 \mathrm{~Hz}, \mathrm{H}-6), 6.79$ (1H, s, H-6'), 6.75 (1H, d, $J=8.1 \mathrm{~Hz}, \mathrm{H}-5), 6.72$ (1H, s, H-2'), 5.58 (1H, d, $J=$ $6.4 \mathrm{~Hz}, \mathrm{H}-7), 4.35$ (1H, d, $J=7.8 \mathrm{~Hz}, \mathrm{H}-1 "), 3.85$ (3H, s, 3'- $\left.-\mathrm{OCH}_{3}\right), 3.82\left(3 \mathrm{H}, \mathrm{s}, 3-\mathrm{OCH}_{3}\right), 3.56(2 \mathrm{H}, \mathrm{t}, J=6.5 \mathrm{~Hz}$, H-9'), 2.62 (2H, m, H-7'), 1.81 (2H, tt, $J=13.1,6.5 \mathrm{~Hz}$, $\left.\mathrm{H}-8^{\prime}\right) ;{ }^{13} \mathrm{C}$ NMR (CD $\left.\mathrm{OD}, 125 \mathrm{MHz}\right): \delta 147.6$ (C-3), 146.1 (C-4), 146.0 (C-4'), 143.8 (C-3'), 135.6 (C-1'), 133.2 (C-1), 128.3 (C-5'), 118.4 (C-6), 116.8 (C-6'), 114.6 (C-5), 112.8 (C-2'), 109.4 (C-2), 102.8 (C-1"), 87.8 (C-7), 76.8 (C-5"), 76.7 (C-3"), 73.7 (C-2"), 70.9 (C-9), 70.2 (C4"), 61.3 (C-6"), 60.8 (C-9'), $55.4\left(3^{\prime}-\mathrm{OCH}_{3}\right), 55.1$ (3$\left.\mathrm{OCH}_{3}\right), 51.5(\mathrm{C}-8), 34.4\left(\mathrm{C}-8^{\prime}\right), 31.5$ (C-7').
Kaempferol-3- $\boldsymbol{O}$ - $\boldsymbol{\beta}$-D-glucopyranoside (4) - Yellow needles; ${ }^{1} \mathrm{H}$ NMR $\left(\mathrm{CD}_{3} \mathrm{OD}, 500 \mathrm{MHz}\right): \delta 8.08(2 \mathrm{H}, \mathrm{d}$, $\left.J=8.8 \mathrm{~Hz}, \mathrm{H}-2^{\prime}, 6^{\prime}\right), 6.91$ (2H, d, $\left.J=8.8 \mathrm{~Hz}, \mathrm{H}-3^{\prime}, 5^{\prime}\right), 6.43$ (1H, s, H-8), 6.23 (1H, s, H-6), 5.28 (1H, d, $J=7.4$ Hz, H$1 ") ;{ }^{13} \mathrm{C}$ NMR (CD $\left.3 \mathrm{OD}, 125 \mathrm{MHz}\right): \delta 178.1$ (C-4), 164.7 (C-7), 161.7 (C-5), 160.1 (C-4'), 157.7 (C-9), 157.1 (C-2), 134.0 (C-3), 130.8 (C-2', 6'), 121.4 (C-1'), 114.7 (C-3', 5'), 104.3 (C-10), 102.7 (C-1"), 98.5 (C-6), 93.6 (C-8), 77.2 (C-5"), 76.7 (C-3"), 74.3 (C-2"), 70.0 (C-4"), 61.2 (C-6").

Nicotiflorin (5) - Yellow needles; ${ }^{1} \mathrm{H}$ NMR $\left(\mathrm{CD}_{3} \mathrm{OD}\right.$, $500 \mathrm{MHz}): \delta 8.09\left(2 \mathrm{H}, \mathrm{d}, J=8.8 \mathrm{~Hz}, \mathrm{H}-2^{\prime}, 6^{\prime}\right), 6.91(2 \mathrm{H}$, d, $J=8.8 \mathrm{~Hz}, \mathrm{H}-3$ ', 5'), $6.42(1 \mathrm{H}, \mathrm{s}, \mathrm{H}-8), 6.23(1 \mathrm{H}, \mathrm{d}$, $J=1.8 \mathrm{~Hz}, \mathrm{H}-6), 5.15$ (1H, d, $J=7.4 \mathrm{~Hz}, \mathrm{H}-1 "), 4.54(1 \mathrm{H}$, d, $\left.J=0.9 \mathrm{~Hz}, \mathrm{H}-1^{\prime \prime \prime}\right), 3.66$ (1H, m, H-2"'), 3.55 (1H, dd, $\left.J=9.5,3.3 \mathrm{~Hz}, \mathrm{H}-3{ }^{\prime \prime \prime}\right), 1.14$ (3H, d, $\left.J=6.2 \mathrm{~Hz}, \mathrm{H}-6{ }^{\prime \prime}\right) ;{ }^{13} \mathrm{C}$ 
Table 2. Inhibitory effects of compounds $\mathbf{1} \mathbf{- 1 2}$ on NO production in LPS-activated BV-2 cells

\begin{tabular}{ccc}
\hline \hline Compound & $\mathrm{IC}_{50}(\mu \mathrm{M})^{a}$ & ${\text { Cell Viability }(\% \text { of LPS })^{b}}^{a}$ \\
\hline 1 & 50.29 & $89.42 \pm 6.36$ \\
2 & $>500$ & $93.08 \pm 5.10$ \\
3 & 26.89 & $109.90 \pm 3.47$ \\
4 & 60.27 & $107.24 \pm 2.72$ \\
5 & $>500$ & $92.35 \pm 7.51$ \\
6 & 108.55 & $112.35 \pm 4.80$ \\
7 & 25.59 & $111.09 \pm 4.11$ \\
8 & $>500$ & $82.11 \pm 2.89$ \\
9 & $>500$ & $91.41 \pm 7.19$ \\
10 & 44.21 & $101.07 \pm 2.86$ \\
11 & 94.38 & $97.45 \pm 11.50$ \\
12 & $>500$ & $86.83 \pm 4.21$ \\
L-NMMA $^{c}$ & 21.40 & $100.14 \pm 2.54$
\end{tabular}

${ }^{\mathrm{a}} \mathrm{IC}_{50}$ value of each compound was defined as the concentration $(\mu \mathrm{M})$ that caused

$50 \%$ inhibition of NO production in LPS-activated BV-2 cells.

${ }^{\mathrm{b}}$ Cell viability after treatment with $20 \mu \mathrm{M}$ of each compound was determined by

MTT assay and is expressed in percentage (\%). The results are averages of three

independent experiments, and the data are expressed as mean \pm SD.

${ }^{\mathrm{c}} \mathrm{L}-\mathrm{NMMA}$ as positive control.

NMR (CD 3 OD, $125 \mathrm{MHz}): \delta 178.0(\mathrm{C}-4), 164.8(\mathrm{C}-7)$, 161.6 (C-5), 160.1 (C-4'), 158.0 (C-9), 157.2 (C-2), 134.1 (C-3), 130.1 (C-2', 6'), 121.4 (C-1'), 114.7 (C-3', 5'), 104.2 (C-10), 103.2 (C-1"), 101.0 (C-1"'), 98.6 (C-6), 93.5 (C8), 76.8 (C-3"), 75.8 (C-5"), 74.4 (C-2"), 72.5 (C-4"'), 70.9 (C-2"'), 70.7 (C-3"'), 70.0 (C-4"), 68.3 (C-5"'), 67.2 (C-6"), 16.5 (C-6"').

p-Hydroxybenzoic acid (6) - White gum; ${ }^{1} \mathrm{H}$ NMR $\left(\mathrm{CD}_{3} \mathrm{OD}, 500 \mathrm{MHz}\right): \delta 7.90\left(2 \mathrm{H}, \mathrm{d}, J=8.8 \mathrm{~Hz}, \mathrm{H}-2^{\prime}, 6^{\prime}\right)$, $6.84\left(2 \mathrm{H}, \mathrm{d}, J=8.8 \mathrm{~Hz}, \mathrm{H}-3^{\prime}, 5^{\prime}\right) ;{ }^{13} \mathrm{C}$ NMR $\left(\mathrm{CD}_{3} \mathrm{OD}, 125\right.$ MHz): $\delta 170.1$ (C-7), 163.3 (C-4), 133.0 (C-2, 6), 122.8 (C-1), $116.0(\mathrm{C}-3,5)$.

ק-Amyrin (7) - Colorless gum; ${ }^{1} \mathrm{H}$ NMR $\left(\mathrm{CDCl}_{3}, 500\right.$ MHz): $\delta 5.19(1 \mathrm{H}, \mathrm{t}, J=3.6 \mathrm{~Hz}, \mathrm{H}-12), 3.22(1 \mathrm{H}, \mathrm{dd}$, $J=11.0,4.6 \mathrm{~Hz}, \mathrm{H}-3), 1.14$ (3H, s, H-27), $1.01(3 \mathrm{H}, \mathrm{s}, \mathrm{H}-$ 26), $0.97(3 \mathrm{H}, \mathrm{s}, \mathrm{H}-23), 0.94(3 \mathrm{H}, \mathrm{s}, \mathrm{H}-25), 0.88(6 \mathrm{H}, \mathrm{s}$, $\mathrm{H}-29,30), 0.84(3 \mathrm{H}, \mathrm{s}, \mathrm{H}-28), 0.80(3 \mathrm{H}, \mathrm{s}, \mathrm{H}-24) ;{ }^{13} \mathrm{C}$ NMR (CDCl $\left.{ }_{3}, 175 \mathrm{MHz}\right): \delta 145.4$ (C-13), 121.9 (C-12), 79.3 (C-3), 55.4 (C-5), 47.8 (C-9), 47.4 (C-18), 47.0 (C19), 41.9 (C-14), 40.0 (C-8), 39.0 (C-4), 38.8 (C-1), 37.3 (C-10), 37.2 (C-22), 34.9 (C-21), 33.5 (C-29), 32.8 (C-7), 32.7 (C-17), 31.3 (C-20), 28.6 (C-28), 28.3 (C-23), 27.3 (C-2), 27.1 (C-16), 26.3 (C-15), 26.2 (C-27), 23.9 (C-30), 23.7 (C-11), 18.5 (C-6), 17.0 (C-26), 15.8 (C-25), 15.7 (C-24).
Table 3. Effects of compounds $\mathbf{1}$ - $\mathbf{1 2}$ on NGF secretion in C6 cells

\begin{tabular}{ccc}
\hline \hline Compound & ${\text { NGF secretion }(\%)^{a}}^{a}$ & ${\text { Cell Viability }(\%)^{b}}^{b}$ \\
\hline 1 & $153.09 \pm 4.66$ & $98.69 \pm 3.69$ \\
2 & $102.90 \pm 2.03$ & $98.46 \pm 11.25$ \\
3 & $105.83 \pm 1.44$ & $97.02 \pm 8.02$ \\
4 & $137.97 \pm 1.34$ & $96.55 \pm 7.38$ \\
5 & $156.88 \pm 8.86$ & $96.81 \pm 17.29$ \\
6 & $149.21 \pm 0.70$ & $86.72 \pm 0.02$ \\
7 & $129.76 \pm 0.91$ & $91.60 \pm 0.70$ \\
8 & $94.75 \pm 8.47$ & $91.65 \pm 1.20$ \\
9 & $157.34 \pm 3.30$ & $95.52 \pm 0.38$ \\
10 & $130.55 \pm 11.41$ & $98.64 \pm 4.12$ \\
11 & $151.05 \pm 2.56$ & $103.14 \pm 0.62$ \\
12 & $104.93 \pm 4.75$ & $83.43 \pm 2.48$ \\
6-Shogaol & $164.74 \pm 1.95$ & $100.34 \pm 7.91$
\end{tabular}

${ }^{\mathrm{a}} \mathrm{C} 6$ cells were treated with $20 \mu \mathrm{M}$ of the compounds. After $24 \mathrm{~h}$, the content of

NGF secreted into C6-conditioned media was measured by ELISA. The level of

secreted NGF is expressed as a percentage of the untreated control (set at $100 \%$ ).

Data are the mean $\pm \mathrm{SD}$ of three independent experiments performed in triplicate.

${ }^{\mathrm{b}}$ Cell viability after treatment with $20 \mu \mathrm{M}$ of each compound was determined by

the MTT assay and is expressed as a percentage (\%). Results are the mean of three

independent experiments, and the data are expressed as the mean \pm SD.

${ }^{\mathrm{c}} 6$-shogaol was used as positive control.

Erythrodiol (8) - Colorless gum; ${ }^{1} \mathrm{H}$ NMR $\left(\mathrm{CDCl}_{3}\right.$, $500 \mathrm{MHz}): \delta 5.20(1 \mathrm{H}, \mathrm{t}, J=3.5 \mathrm{~Hz}, \mathrm{H}-12), 3.55(1 \mathrm{H}, \mathrm{d}$, $J=10.9 \mathrm{~Hz}, \mathrm{H}-28 \mathrm{a}), 3.22(1 \mathrm{H}, \mathrm{d}, J=10.9 \mathrm{~Hz}, \mathrm{H}-28 \mathrm{~b})$, $3.22(1 \mathrm{H}, \mathrm{d}, J=10.9 \mathrm{~Hz}, \mathrm{H}-3), 1.17$ (3H, s, H-27), 1.00 (3H, s, H-23), 0.95 (3H, s, H-26), 0.94 (3H, s, H-25), 0.89 (3H, s, H-29), 0.88 (3H, s, H-30), 0.79 (3H, s, H-24); ${ }^{13} \mathrm{C}$ NMR (CDCl, $175 \mathrm{MHz}): \delta 144.2$ (C-13), 122.4 (C-12), 79.0 (C-3), 69.7 (C-28), 55.2 (C-5), 47.6 (C-9), 46.5 (C19), 42.4 (C-18), 41.7 (C-14), 39.8 (C-8), 38.9 (C-4), 38.6 (C-1), 37.0 (C-10, 17), 34.1 (C-21), 33.2 (C-29), 32.6 (C7), 31.0 (C-20), 30.9 (C-22), 28.0 (C-23), 27.2 (C-2), 25.9 (C-27), 25.6 (C-15), 23.6 (C-11), 23.5 (C-30), 22.0 (C16), 18.3 (C-6), 16.7 (C-26), 15.6 (C-24), 15.5 (C-25).

$\boldsymbol{\alpha}$-Spinasterol (9) - White needles; ${ }^{1} \mathrm{H}$ NMR $\left(\mathrm{CDCl}_{3}\right.$, $500 \mathrm{MHz}): \delta 5.16(1 \mathrm{H}, \mathrm{dd}, J=15.7,8.7 \mathrm{~Hz}, \mathrm{H}-22), 5.03$ $(1 \mathrm{H}, \mathrm{dd}, J=15.2,8.6 \mathrm{~Hz}, \mathrm{H}-23), 3.60(1 \mathrm{H}, \mathrm{tt}, J=10.9$, $4.5 \mathrm{~Hz}, \mathrm{H}-3), 1.03(3 \mathrm{H}, \mathrm{d}, J=6.6 \mathrm{~Hz}, \mathrm{H}-3), 0.85(3 \mathrm{H}, \mathrm{d}$, $J=6.5 \mathrm{~Hz}, \mathrm{H}-26), 0.56$ (3H, s, H-18); ${ }^{13} \mathrm{C}$ NMR $\left(\mathrm{CDCl}_{3}\right.$, $175 \mathrm{MHz}): \delta 139.8(\mathrm{C}-8), 139.4(\mathrm{C}-22), 129.7$ (C-23), 117.7 (C-7), 71.3 (C-3), 56.1 (C-17), 55.4 (C-14), 51.5 (C-24), 49.7 (C-9), 43.5 (C-13), 41.0 (C-20), 40.5 (C-5), 39.7 (C-12), 38.2 (C-4), 37.4 (C-1), 34.4 (C-10), 32.1 (C- 
25), 31.7 (C-2), 29.9 (C-6), 28.7 (C-16), 25.6 (C-28), 23.2 (C-15), 21.8 (C-11), 21.6 (C-26), 21.3 (C-21), 19.2 (C27), 13.2 (C-19), 12.5 (C-29), 12.3 (C-18).

29-Nor-20-oxolupeol (10) - White gum; ${ }^{1} \mathrm{H}$ NMR $\left(\mathrm{CDCl}_{3}, 500 \mathrm{MHz}\right): \delta 3.19(1 \mathrm{H}, \mathrm{dd}, J=11.4,4.8 \mathrm{~Hz}, \mathrm{H}-$ 3), $2.58(1 \mathrm{H}, \mathrm{td}, J=11.3,6.0 \mathrm{~Hz}, \mathrm{H}-19), 2.15(3 \mathrm{H}, \mathrm{s}, \mathrm{H}-$ 30), 1.02 (3H, s, H-26), 0.97 (6H, s, H-23, 27), $0.83(3 \mathrm{H}$, s, H-25), $0.78(3 \mathrm{H}, \mathrm{s}, \mathrm{H}-28), 0.76(3 \mathrm{H}, \mathrm{s}, \mathrm{H}-24) ;{ }^{13} \mathrm{C}$ NMR $\left(\mathrm{CDCl}_{3}, 175 \mathrm{MHz}\right): \delta 213.1(\mathrm{C}-20), 79.2(\mathrm{C}-3)$, 55.5 (C-5), 52.9 (C-19), 50.5 (C-9), 49.9 (C-18), 43.3 (C14), 42.9 (C-17), 41.0 (C-8), 40.1 (C-22), 39.1 (C-4), 38.9 (C-1), 37.4 (C-10), 37.3 (C-13), 35.2 (C-16), 34.4 (C-7), 29.4 (C-30), 28.2 (C-23), 27.9 (C-21), 27.6 (C-15), 27.5 (C-2), 27.4 (C-12), 21.1 (C-11), 18.5 (C-6), 18.2 (C-28), 16.3 (C-25), 16.1 (C-26), 15.6 (C-24), 14.7 (C-27).

Lupenone (11) - Colorless gum; ${ }^{1} \mathrm{H}$ NMR $\left(\mathrm{CDCl}_{3}\right.$, $500 \mathrm{MHz}): \delta 4.69(1 \mathrm{H}, \mathrm{d}, J=2.4 \mathrm{~Hz}, \mathrm{H}-29 \mathrm{a}), 4.57(1 \mathrm{H}$, dd, $J=2.4,1.4 \mathrm{~Hz}, \mathrm{H}-29 \mathrm{~b}), 1.69$ (3H, brs, H-30), 1.07 (6H, s, H-24, 26), 1.03 (3H, s, H-23), 0.96 (3H, s, H-27), 0.93 (3H, s, H-25), 0.80 (3H, s, H-28); ${ }^{13} \mathrm{C}$ NMR $\left(\mathrm{CDCl}_{3}\right.$, $175 \mathrm{MHz}): \delta 218.2$ (C-3), 150.9 (C-20), 109.4 (C-29), 54.9 (C-5), 49.8 (C-9), 48.2 (C-18), 47.9 (C-19), 47.3 (C4), 43.0 (C-14), 42.9 (C-17), 40.8 (C-8), 40.0 (C-22), 39.6 (C-2), 38.2 (C-13), 36.9 (C-10), 35.5 (C-16), 34.1 (C-1), 33.6 (C-7), 29.8 (C-21), 27.4 (C-15), 26.6 (C-23), 25.1 (C-12), 21.5 (C-11), 21.0 (C-24), 19.7 (C-6), 19.3 (C-30), 18.0 (C-28), 16.0 (C-25), 15.8 (C-26), 14.5 (C-27).

Lupeol (12) - White gum; ${ }^{1} \mathrm{H} \mathrm{NMR}\left(\mathrm{CDCl}_{3}, 500 \mathrm{MHz}\right)$ : $\delta 4.69(1 \mathrm{H}, \mathrm{d}, J=2.3 \mathrm{~Hz}, \mathrm{H}-29 \mathrm{a}), 4.57$ (1H, dd, $J=2.4$, $1.4 \mathrm{~Hz}, \mathrm{H}-29 \mathrm{~b}), 3.19$ (1H, dd, $J=11.4,4.9 \mathrm{~Hz}, \mathrm{H}-3), 2.37$ $(1 \mathrm{H}, \mathrm{td}, J=11.1,5.8 \mathrm{~Hz}, \mathrm{H}-19), 1.68$ (3H, brs, H-30), 1.03 (3H, s, H-26), 0.97 (3H, s, H-27), 0.95 (3H, s, H-23), 0.83 (3H, s, H-25), 0.79 (3H, s, H-24), 0.76 (3H, s, H-28); ${ }^{13} \mathrm{C}$ NMR $\left(\mathrm{CDCl}_{3}, 175 \mathrm{MHz}\right): \delta 151.2(\mathrm{C}-20), 109.4(\mathrm{C}-$ 29), 79.1 (C-3), 55.6 (C-5), 50.5 (C-9), 48.7 (C-19), 48.3 (C-18), 43.2 (C-17), 43.1 (C-14), 41.1 (C-8), 40.3 (C-22), 39.0 (C-1, 4), 38.3 (C-13), 37.2 (C-10), 35.6 (C-16), 34.4 (C-7), 30.1 (C-21), 28.4 (C-23), 27.9 (C-15), 27.7 (C-2), 25.4 (C-12), 21.3 (C-11), 19.7(C-30), 18.6 (C-6), 18.3(C28), 16.5 (C-25), 16.3 (C-26), 15.7 (C-24), 14.7 (C-27).

Measurement of NO Production and Cell Viability Murine microglial BV-2 cells were plated into a 96-well plate $\left(3 \times 10^{4}\right.$ cells $/$ well). After $24 \mathrm{~h}$, cells were pretreated with samples for $30 \mathrm{~min}$ and then stimulated with $100 \mathrm{ng} /$ $\mathrm{mL}$ of LPS for another $24 \mathrm{~h}$. Nitrite, a soluble oxidation product of NO, was measured in culture media using the Griess reaction. The supernatant $(50 \mu \mathrm{L})$ was harvested and mixed with an equal volume of Griess reagent $(1 \%$ sulfanilamide, $0.1 \% \mathrm{~N}$-1-naphthylethylenediamine dihydrochloride in $5 \%$ phosphoric acid). After $10 \mathrm{~min}$, the absorbance at $540 \mathrm{~nm}$ was measured using a microplate reader. Sodium nitrite was used as a standard to calculate the $\mathrm{NO}_{2}{ }^{-}$concentration. Cell viability was tested by the 3[4,5-dimethylthiazol-2-yl]-2,5-diphenyl-tetrazolium bromide (MTT) assay. $N^{\mathrm{G}}$-Mono-methyl-L-arginine was evaluated as a positive control.

NGF and cell viability assays - C6 glioma cells were used to measure NGF release into the medium. C6 cells were purchased from the Korean Cell Line Bank and maintained in DMEM supplemented with 10\% FBS and $1 \%$ penicillin-streptomycin in a humidified incubator with $5 \% \mathrm{CO}_{2}$. C6 cells were seeded into 24-well plates $(1 \times$ $10^{5}$ cells/well). After $24 \mathrm{~h}$, the cells were treated with DMEM containing $2 \%$ FBS and $1 \%$ penicillin-streptomycin with $20 \mu \mathrm{M}$ of each sample for one day. The cell viability of the C6 cells was tested via an MTT assay. 6-shogaol was evaluated as a positive control.

\section{Result and Discussion}

The structures of compounds $\mathbf{2}$ - $\mathbf{1 2}$ were determined by comparison of their spectroscopic data with those in the literatures to be $1 \beta, 2 \beta, 4 \beta$-triol-1,2,3,4-tetrahydronaphthalene (2) ${ }^{11}$ (7R,8S)-dihydrodehydrodiconiferyl alcohol-9-O- $\beta$ D-glucopyranoside (3), ${ }^{12}$ kaempferol-3- $O-\beta$-D-glucopyranoside (4) ${ }^{13}$ nicotiflorin (5), ${ }^{14} p$-hydroxybenzoic acid (6), ${ }^{15}$ $\beta$-amyrin (7) ${ }^{16}$ erythrodiol (8), ${ }^{17} \alpha$-spinasterol (9), ${ }^{18} 29$-nor20-oxolupeol (10), ${ }^{19}$ lupenone (11), ${ }^{20}$ and lupeol (12). ${ }^{21}$

Compound 1 was obtained as a colorless gum. The molecular formula was determined to be $\mathrm{C}_{10} \mathrm{H}_{12} \mathrm{O}_{3}$, based on molecular ion peak $[\mathrm{M}+\mathrm{H}]^{+}$at $m / z 181.0865$ (calcd for $\mathrm{C}_{10} \mathrm{H}_{13} \mathrm{O}_{3}, 118.0865$ ) in the positive-ion HR-ESI-MS. The IR spectrum of 1 indicated the presence of hydroxy group $\left(3348 \mathrm{~cm}^{-1}\right)$. The ${ }^{1} \mathrm{H}$ NMR spectrum of 1 showed the presence of a 1,2-disubstituted aromatic ring protons at $\delta_{\mathrm{H}}$ $7.56(1 \mathrm{H}, \mathrm{d}, J=7.3 \mathrm{~Hz}), 7.41(1 \mathrm{H}, \mathrm{dd}, J=7.3,1.5 \mathrm{~Hz})$, $7.34(1 \mathrm{H}, \mathrm{td}, J=7.3,1.5 \mathrm{~Hz})$, and $7.29(1 \mathrm{H}, \mathrm{td}, J=7.3$, $1.5 \mathrm{~Hz})$, three oxygenated proton signals at $\delta_{\mathrm{H}} 4.89(1 \mathrm{H}$, m), $4.41(1 \mathrm{H}, \mathrm{d}, J=7.3 \mathrm{~Hz})$, and $4.09(1 \mathrm{H}$, ddd, $J=10.1$, $7.3,3.4 \mathrm{~Hz}$ ) and one methylene proton signal at $\delta_{\mathrm{H}} 2.21$ $(1 \mathrm{H}, \mathrm{ddd}, J=13.5,4.7,3.4 \mathrm{~Hz})$, and $2.12(1 \mathrm{H}$, ddd, $J=13.5,10.1,4.7 \mathrm{~Hz})$. The ${ }^{13} \mathrm{C}$ NMR spectrum displayed 10 carbon signals, including aromatic carbon signals at $\delta_{\mathrm{C}}$ 137.6, 137.5, 128.1, 127.6, 127.4, and 127.1, three oxygenated carbon signals at $\delta_{\mathrm{C}} 73.6,68.9$, and 66.6 , one methylene carbon signal at $\delta_{\mathrm{C}} 37.1$. These NMR data were similar to $1 \alpha, 2 \alpha, 4 \beta$-triol-1,2,3,4-tetrahydronaphthalene, except for chemical shifts and coupling constants of methine protons. ${ }^{22}$ The location of hydroxyl groups was confirmed by ${ }^{1} \mathrm{H}-{ }^{1} \mathrm{H}$ COSY correlations and HMBC data 

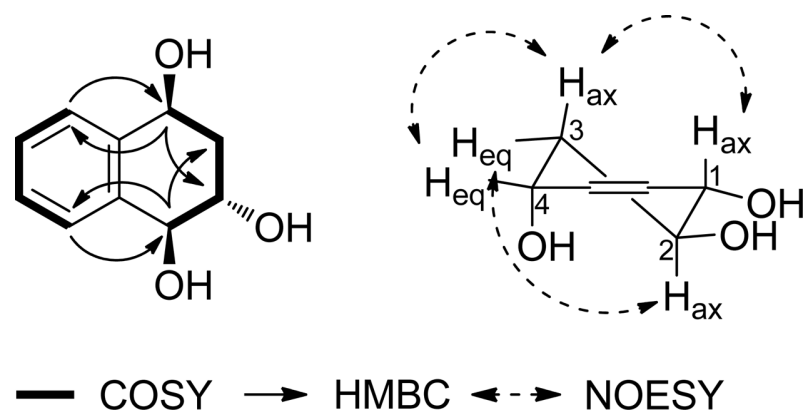

Fig. 2. Key ${ }^{1} \mathrm{H}-{ }^{1} \mathrm{H}$ COSY $(\longrightarrow), \operatorname{HMBC}(\longrightarrow)$ and NOESY $(+\cdots \cdots \rightarrow)$ correlations of $\mathbf{1}$.

(Fig. 2). The relative configuration of 1 established based on coupling constants $\left({ }^{3} J_{1,2}=7.3 \mathrm{~Hz},{ }^{3} J_{2,3}=10.1,3.4 \mathrm{~Hz}\right.$, and ${ }^{3} J_{3,4}=4.7 \mathrm{~Hz}$ ) of ${ }^{1} \mathrm{H}-\mathrm{NMR}$ spectra, leading oxygenated protons at $\mathrm{C}-1, \mathrm{C}-2$, and $\mathrm{C}-4$ to be as axial, axial, and equatorial positions, respectively (Fig. 2). ${ }^{22,23}$ In the NOESY spectrum, correlations of $\mathrm{H}-3_{\mathrm{ax}}$ with $\mathrm{H}-1$ and $\mathrm{H}-$ 4 indicated that $\mathrm{OH}$ groups at $\mathrm{C}-1$ and $\mathrm{C}-4$ were to be in the same orientation, and cross peak $\mathrm{H}-2 / \mathrm{H}-3_{\text {eq }}$ showed that $\mathrm{OH}$ groups at $\mathrm{C}-1$ and $\mathrm{C}-2$ were to be in the opposite orientation (Fig. 2). Thus, the structure of $\mathbf{1}$ was established as $1 \beta, 2 \alpha, 4 \beta$-triol-1,2,3,4-tetrahydronaphthalene.

The isolated compounds (1-12) were evaluated for their inhibitory effects on NO production in LPS-activated murine microglial cells and their effects on NGF secretion from C6 glioma cells. Among them, compounds 3, 7, and 10 reduced NO levels in LPS-activated murine microglial BV-2 cells with $\mathrm{IC}_{50}$ values of $26.89,25.59$, and 44.21 $\mu \mathrm{M}$, respectively. Compounds 1, 5, and 9 upregulated NGF secretion to $153.09 \pm 4.66,156.88 \pm 8.86$, and 157.34 $\pm 3.30 \%$, respectively.

\section{Acknowledgments}

This research was supported by the Basic Science Research Program through the National Research Foundation of Korea (NRF) funded by the Ministry of Education, Science and Technology (2012R1A5A2A 28671860). We are thankful to the Korea Basic Science Institute (KBSI) for the NMR and mass spectrum data.

\section{References}

(1) Meenu, B.; Neeraja, E. D.; Rejimon, G.; Varghese, A. J. Chem. Pharm. Res. 2015, 7, 16-21.

(2) Ding, Z. S.; Jiang, F. S.; Chen, N. P.; Lv, G. Y.; Zhu, C. G. molecules 2008, 13, 220-229.

(3) Kang, S. N.; Goo, Y. M.; Yang, M. R.; Ibrahim, R. I. H.; Cho, J. H.; Kim, I. S.; Lee, O. H. molecules 2013, 18, 6356-6365.

(4) Shoji, N.; Umeyama, A.; Yosikawa, K.; Nagai, M. Arihara, S. Phytochemistry, 1994, 37, 1437-1441.

(5) Panichayupakaranant, P.; Noguchi, H.; De-Eknamkul, W.; Sankawa. U. Phytochemistry, 1995, 40, 1141-1143.

(6) Li, Q.; Zhang, X.; Cao, J.; Guo, Z.; Lou, Y.; Ding, M.; Zhao, Y. Fitoterapia 2015, 105, 234-239.

(7) Ishiguro, K.; Ohira, Y.; Oku, H. J. Nat. Prod. 1998, 61, 1126-1129.

(8) Imam, M. Z.; Nahar, N.; Akter, S.; Rana, M. S. J. Ethnopharmacol. 2012, 142, 804-810.

(9) Kim, C. S.; Subedi, L.; Kim, S. Y.; Choi, S. U.; Choi, S. J.; Son, M. W.; Kim, K. H.; Lee, K. R. Phytochem. lett. 2015, 14, 215-220.

(10) Kim, C. S.; Bae, M.; O, J.; Subedi, L.; Suh, W. S.; Choi, S. Z.; Son, M. W.; Kim, S. Y.; Choi, S. U.; Oh, D. C.; Lee, K. R. J. Nat. Prod. 2017, 80, 471-478.

(11) Rashid, A.; Read, G. J. Chem. Soc. 1969, 15, 2053-2058.

(12) Zhao, X. C.; Du. J. L.; Xie, Y. G.; Zhang, Y.; Jin, H. Z. Chem. Nat. Compd. 2018, 54, 556-558.

(13) Jayasinghe, U. L. B.; Balasooriya, B. A. I. S.; Bandara, A. G. D.; Fujimoto, Y. Nat. Prod. Res. 2004, 18, 499-502.

(14) Park, S. Y.; Kim, S. J.; Lee, S. Y.; Bae, K. H.; Kang, S. S. Nat. Prod. Sci. 2008, 14, 281-288.

(15) Rho, T. W.; Yoon, K. D. Nat. Prod. Sci. 2017, 23, 253-257.

(16) Wang, H.; Tian, X.; Chen, Y. Z. J. Chin. Chem. Soc. 2002, 49, 433436.

(17) Ge, Y. C.; Zhang, H. J.; Lei, J. X.; Wang, K. W. Chem. Nat. Compd. 2018, 54, 600-602.

(18) Choi, S. Z.; Lee, S. O.; Choi, S. U.; Lee, K. R. Arch. Pharm. Res. 2003, 26, 521-525.

(19) Kotowicz, C.; Catalan, C. A. N.; Griffin, C. L.; Herz, W. Biochem. Syst. Ecol. 2005, 33, 737-742.

(20) Puapairoj, P.; Naengchomnong, W.; Kijjoa, A.; Pinto, M. M.; Pedro, M.; Nascimento, M. S.; Silva, A. M.; Herz, W. Planta Med. 2005, $71,208-213$.

(21) Fotie, J.; Bohle, D. S.; Leimanis, M. L.; Georges, E.; Rukunga, G.; Nkengfack, A. E. J. Nat. Prod. 2006, 69, 62-67

(22) Chen, X. M; Qian, S. H; Feng, F. Chine. Chem. Lett. 2010, 21, 440-442.

(23) Naemura, K.; Wakebe, T.; Hirose, K.; Tobe, Y. Tetrahedron: Asymmetry 1997, 15, 2585-2595.

Received November 27, 2018 Revised December 12, 2018 Accepted December 12, 2018 